\title{
KSNM/KSID/KOSHIC Guidance for Nuclear Medicine Department Against the Coronavirus Disease 2019 (COVID-19) Pandemic
}

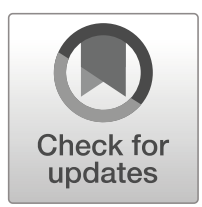

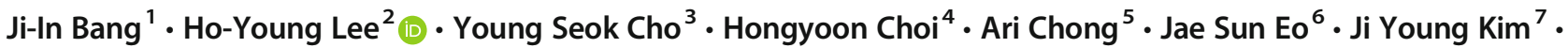 \\ Tae Sung Kim ${ }^{8} \cdot$ Hyun-Woo Kwon ${ }^{9}$ • Eun Jeong Lee ${ }^{10}$. Eun Seong Lee ${ }^{6} \cdot$ Hye Lim Park ${ }^{11}$. Soo Bin Park ${ }^{12}$. \\ Hye-kyung Shim ${ }^{13} \cdot$ Bong-II Song $^{14} \cdot$ Ik Dong Yoo ${ }^{15} \cdot$ Kyung Jae Lee $^{2} \cdot$ Hong Jae Lee $^{4} \cdot$ Su Ha Han ${ }^{16} \cdot$ Jin Seo Lee $^{17}$. \\ Jung Mi Park ${ }^{18}$. Sung Hoon Kim ${ }^{19}$
}

Received: 2 June 2020 / Revised: 20 July 2020 / Accepted: 23 July 2020 / Published online: 11 August 2020

(C) Korean Society of Nuclear Medicine 2020

\begin{abstract}
The dramatic spread of Coronavirus Disease 2019 (COVID-19) has profound impacts on every continent and life. Due to humanto-human transmission of COVID-19, nuclear medicine staffs also cannot escape the risk of infection from workplaces. Every staff in the nuclear medicine department must prepare for and respond to COVID-19 pandemic which tailored to the characteristics of our profession. This article provided the guidance prepared by the Korean Society of Nuclear Medicine (KSNM) in cooperation with the Korean Society of Infectious Disease (KSID) and Korean Society for Healthcare-Associated Infection Control and Prevention (KOSHIC) in managing the COVID-19 pandemic for the nuclear medicine department. We hope that this guidance will support every practice in nuclear medicine during this chaotic period.
\end{abstract}

Keywords Coronavirus $\cdot$ COVID-19 $\cdot$ Nuclear medicine $\cdot$ Prevention and control $\cdot$ Practice guideline

Ho-Young Lee

debobkr@gmail.com

1 Department of Nuclear Medicine, CHA Bundang Medical Center, CHA University of Medicine (Professor), Pocheon, Republic of Korea

2 Department of Nuclear Medicine, Seoul National University Bundang Hospital (Professor), Seongnam, Gyeonggi-do, Republic of Korea

3 Department of Nuclear Medicine, Samsung Medical Center, Seoul, Republic of Korea

4 Department of Nuclear Medicine, Seoul National University Hospital, Seoul, Republic of Korea

5 Department of Nuclear Medicine, Chosun University Hospital, Gwangju, Republic of Korea

6 Department of Nuclear Medicine, Korea University Guro Hospital, Seoul, Republic of Korea

7 Department of Nuclear Medicine, Hanyang University Guri Hospital, Seoul, Republic of Korea

8 Department of Nuclear Medicine, National Cancer Center, Goynag, Republic of Korea

9 Department of Nuclear Medicine, Korea University Anam Hospital, Seoul, Republic of Korea
10 Department of Nuclear Medicine, Seoul Medical Center, Seoul, Republic of Korea

11 Division of Nuclear Medicine, Department of Radiology, Eunpyeong St. Mary's Hospital, College of Medicine, The Catholic University of Korea, Seoul, Republic of Korea

12 Department of Nuclear Medicine, Soonchunhyang University Seoul Hospital, Bucheon, Republic of Korea

13 Department of Nuclear Medicine, Inje University Haeundae Paik Hospital, Busan, Republic of Korea

14 Department of Nuclear Medicine, Keimyung University Dongsan Medical Center, Daegu, Republic of Korea

15 Department of Nuclear Medicine, Soonchunhyang University Cheonan Hospital, Cheonan, Republic of Korea

16 Department of Nursing, Soonchunhyang University Bucheon Hospital, Bucheon, Republic of Korea

17 Division of Infectious Disease, Department of Internal Medicine, Kangdong Sacred Heart Hospital, Hallym University, Chuncheon, Republic of Korea

18 Department of Nuclear Medicine, Soonchunhyang University Bucheon Hospital, Bucheon, Republic of Korea

19 Korean Society of Nuclear Medicine Quality Control Committee, Bucheon, Republic of Korea 


\section{Introduction}

Since the first reports of Coronavirus Disease 2019 (COVID19) in Wuhan, China, the infection had spread worldwide rapidly and COVID-19 has reached pandemic levels. In South Korea, since its outbreak in February 2020, COVID19 has affected profoundly every aspect of communities. The human-to-human transmission of COVID-19 provides challenges for all healthcare facilities and healthcare providers.

In the face of the COVID-19 pandemic, the Korean Society of Nuclear Medicine (KSNM), Korean Society of Infectious Disease (KSID), and Korean Society for HealthcareAssociated Infection Control and Prevention (KOSHIC) have prepared the guidance for the nuclear medicine department to minimize confusion and ensure that nuclear medicine physicians and technicians continue to provide their services while protecting the patients and workers and preventing the transmission of the virus. The Quality Control Committee of KSNM reviewed several reports and recommendations previously published by the European Association of Nuclear Medicine (EANM) [1], Society of Nuclear Medicine and Molecular Imaging (SNMMI), American Society of Nuclear Cardiology (ASNC) [2], International Atomic Energy Agency (IAEA), and others [3-6]. This guidance is basically in compliance with the COVID-19 guidelines of the Korea Centers for Disease Control and Prevention (KCDC) [7-10]. Finally, this document was prepared in cooperation with KSID and KOSHIC. KSNM emphasize that this guidance must be considered in the context of following the state and hospital infection control policies and flexibly applied according to changes in circumstances and evidence.

\section{General Principles During COVID-19 Pandemic}

1. In a pandemic situation, such as COVID-19, if necessary, the condition of the scheduled patient can be checked in advance to adjust the examination schedule.

2. Non-urgent, elective studies or therapy should be postponed in COVID-19-confirmed or COVID-19-suspected patients. Rescheduling the studies/therapy must be done in a discussion with the referring clinicians.

3. Only urgent studies or therapy could be performed in COVID-19-confirmed or COVID-19-suspected patients whenever clinically appropriate. The priority of study/ therapy should be based on a case-by-case in-depth discussion between nuclear medicine physicians and referring clinicians. In case of performing the urgent studies/ therapy, consult with the infection control offices of each institution to comply with the infection control rules of own.
4. COVID-19-suspected patients should undergo COVID19 testing before performing the studies/therapy.

5. Lung ventilation scan should not be performed in any COVID-19-confirmed or COVID-19-suspected patients.

6. Low-dose radioiodine therapy may be considered in case of acute hyperthyroidism patients who are unable to tolerate anti-thyroid medications. As low-dose radioiodine therapy (lower than 1.2 GBq of I-131) can be performed in an outpatient setting in South Korea, COVID-19infected patient can be administrated low-dose radioiodine in the isolation room or negative pressure room without any additional monitoring related to radioiodine therapy.

\section{Consideration During the Study/Therapy}

1. Patient transportation

- Scheduling COVID-19-confirmed or COVID-19suspected patient as last study of the day to prevent cross-infection in the nuclear medicine department.

- Ensure that other patients or caregivers should not access the nuclear medicine department to minimize the exposure to COVID-19 patient during the study/ therapy.

- Transfer the COVID-19-infected patient to the nuclear medicine department using negative pressure transport bag to minimize exposure and contact to droplet.

- COVID-19 patients should wear masks at all times of procedures. If necessary, add gowns, gloves, etc.

2. Devices and scanner management

- Mainly use disposable instruments or items. Do not reuse disposable items such as oxygen masks, nasal prongs, suction tubes, or suction lines. The protocol for reusable devices is as follows:

\section{1) Cleaning}

1) After use, the equipment contaminated with blood, body fluids, secretions, and feces should be delivered to a washing room with care not to contaminate the surrounding environment.

2) The washing place should be separated from the space used for cleaning other items or other patients. After immersing the contaminated equipment in a washing space, wash the product carefully to avoid splashing.

3) Wash enough to remove blood, body fluids, secretions, and feces from remaining. 
4) Staff undertaking cleaning should wear KF94 or N95 masks, long-sleeved waterproof gowns, goggles or face shields, hats, shoe covers or rubber boots, and double gloves (outer gloves are rubber gloves).

2) Disinfection and sterilization

1) Depending on the risk level of the device (according to the Spaulding Classification of medical equipment/devices), non-critical devices require low-level disinfection, semicritical devices require high-level disinfection/sterilization, and critical devices must be sterilized.

2) Disinfectants and sterilization methods by device classification should be followed in accordance with the notification of the Ministry of Health and Welfare (2017-101, 4).

3) Be sure to check the disinfectant manufacturer's recommendations. The recommended disinfection process, such as dilution and application time of disinfectant, and the effective period and concentration of disinfectant are strictly followed.

\section{Laboratory and scan room management}

- Only the minimum number of staffs should be placed in the nuclear medicine department. All participating staffs should wear appropriate personal protective equipment (PPE): eye protection with goggles or face shield, medical protective masks (N95/KF94, or equivalent respirator), disposable latex gloves, disposable gown, disposable shoe covers, etc.

- Cover the scanner couch or other equipment with a plastic cover to prevent contamination.

- Every effort should be made to minimize the COVID-19 exposure to medical staff during injection of radiopharmaceuticals.

- Select the protocol with the shortest duration of uptake time and scan time to minimize the time spent by the COVID-19 patient in the department.

- In case of studies requiring an uptake phase, COVID-19 patients should be waiting in separate space. If possible, COVID-19 patients wait in negative pressure transport bag. If negative pressure transport bag is not available, use bed or stretcher in waiting room with disposable cover. Consider using standard radiopharmaceutical dose to shorten the procedure time.

- After the completion of image acquisition, the scan room and patient's space (area) should be disinfected according to the standard protocol.

- After image acquisition, remove the plastic cover of the scanner and disinfect the scanner surface.

- $\quad$ Remove and discard PPE adequately when leaving the camera room or care area, and immediately perform hand hygiene.
- In case of performing the radiolabeling of the COVID-19 patients' blood products, every process with infectious materials (opening/stirring/mixing/ dispensing COVID-19 patient's blood sample, radiolabeling, etc.) should be done in class II biosafety cabinet according to the Biosafety Level 2 Regulation. Disinfection of laboratory with proper disinfectants $(70 \%$ ethanol, $0.5 \%$ hydrogen peroxide, or 1000 ppm sodium hypochlorite) should be done.

- Used PPE and disposable covers are removed with caution not to contaminate the clean area and disposed in a container for biosafety waste.

4. Employee management

- All employees should be trained in the prevention and management of COVID-19 infection and adhere to the rules of infection prevention.

- Considering the skill level, fatigue, etc. of the working staff, sufficient personnel are allocated to secure them.

- Priority from exemption is given to employees with high-risk underlying diseases such as diabetes mellitus, chronic obstructive pulmonary disease (COPD), end-stage renal disease (ESRD), chronic cardiac disease, etc. or pregnant women.

5. Cleaning and environmental management

- General principle

1) Personnel responsible for cleaning or disinfection should complete the infection prevention education.

2) Employees should wear PPE (KF94 or N95 respirators, full-body protective clothing or aprons, goggles or face shields, shoe covers or rubber boots, double gloves (outer gloves are rubber gloves)) when cleaning or disinfecting.

3) If there are organic substances on the surface of the environment, it cannot be properly disinfected. Therefore, wipe the surface before disinfecting the environment.

4) In order to prevent the possibility of microbial spraying, cleaning should be performed using a cleaning solution or a mop moistened with a disinfectant, rather than a cleaning method using a broom or a vacuum cleaner.

5) Instead of spraying disinfectants, thoroughly clean the surface of the environment using a clean towel moistened with the disinfectant or a commercially available disinfecting tissue (towel). 
6) Use cleaning tools as disposable as possible or exclusively. However, when the cleaning tool is reused, the used cleaning tool is sterilized using an appropriate disinfectant and then dried and stored.

- Disinfection of a patient's space (area)

1) In the case of the space (area) used by the patient, mark the place where contamination was confirmed before cleaning and disinfecting the surface and seal the contaminated object (to prevent others from being exposed).

2) Ventilation before, during, and after cleaning/ disinfection (disinfection after ventilation for $1 \mathrm{~h}$ based on 6 air cycles per hour).

3) Wear PPE. Wipe with a cloth (cloth, etc.) wet with the diluted disinfectant. Wipe the touched wall surface and all frequently used areas, and keep it for at least $10 \mathrm{~min}$. After then, wipe the surface with a cloth dampened with clean water (cloth, etc.)

- (Resumption of use) Consider the characteristics of each type of disinfectant used and the purpose of the facility.

* After disinfection, the virus is killed, but the decision at the time of resumption of use cannot be applied in batch due to different characteristics of disinfectants, so it is necessary to consider the precautions for each product.

- For details on disinfecting methods such as surface disinfection and washing, refer to "Disinfection Guidelines to Prevent the Spread of COVID-19 at Public and Multi-purpose Facilities. 3rd edition.” Refer to the method of disinfecting the patient space (area).

- (Select an environmental disinfectant) Select an approved or declared disinfectant by the Ministry of Environment, and follow the usage, usage, and precautions for each product.

- Disinfectant list of the Ministry of Environment (http://ecolife.me.go.kr)

* Precautions when using environmental disinfectants:

1) Select the disinfectant after confirming information such as approval from the Ministry of Environment and Environment.

2) When using environmental sterilizers, make sure to follow the manufacturer's recommendations, such as checking the expiration date, safe usage for each product, and precautions and preparing the diluent according to the manufacturer's instructions.

3) The disinfecting method of spraying/injecting disinfectant is not applied to surface disinfection because it causes aerosol infection, increased risk of inhalation, and the range of contact between the disinfectant and the surface is insufficient, so the disinfecting effect is insufficient.

4) Disinfectant hazard information must be checked and used carefully.

5) Do not mix different disinfectants. Do not place near flammable materials. Disinfectant should be used in a well-ventilated area.

6) As the disinfection effect may decrease over time, dilute as much as necessary and use it immediately. Do not store the remaining amount, and discard it immediately.

6. Laundry management

- Store clean laundry in a separate space.

- Employees handling laundry should be trained to prevent infection.

- Employees handling contaminated laundry should wear PPE (N95 masks or equivalent respiratory protection, gowns, gloves, overshoes, etc.) and perform hand hygiene after removing PPE.

- The laundry used for the patient is disposed of according to the relevant regulations (see Waste Management Act, Medical Institution Laundry Management Rules, etc.).

- Thoroughly ensure that pathogens are not exposed to personnel handling the laundry or surrounding environment during the entire process of collecting, transporting, and washing laundry.

7. Waste management

- Waste related to COVID-19 patients is managed by the rules of hospital infectious control policy.

- Sharp tools, such as needles or blades, are collected in containers for impervious and non-permanent waste, and containers should be stored in the place where the items are used.

- Simple infectious waste contaminated or possibly contaminated with COVID-19 patients' sample is autoclaved and discarded. Radioactive waste should be discarded in compliance with national regulation with caution not to contaminate the staff or area.

\section{Conclusion}

Considering that outbreaks of novel viruses have been periodically appearing these days, nuclear medicine staffs should get used to guidance and policies for infectious disease in working 
place to protect patients, worker themselves, and furthermore valuable medical resources. Basically, this guidance can be applied in case of any other human-to-human transmission disease for operating the nuclear medicine department. Also, always bear in mind the rapid change in the situation; this guidance should be used in conjunction with the current government and local hospital policies.

\section{Compliance with Ethical Standards}

Conflict of Interest Ji-In Bang, Ho-Young Lee, Young Seok Cho, Hongyoon Choi, Ari Chong, Jae Sun Eo, Ji Young Kim, Tae Sung Kim, Hyun-Woo Kwon, Eun Jeong Lee, Eun Seong Lee, Hye Lim Park, Soo Bin Park, Hye-kyung Shim, Bong-Il Song, Ik Dong Yoo, Kyung Jae Lee, Hong Jae Lee, Su Ha Han, Jin Seo Lee, Jung Mi Park, and Sung Hoon Kim declare that they have no conflict of interest.

Ethical Approval This work does not contain any studies with human participants or animals performed by any of the authors.

Informed Consent Not applicable.

\section{References}

1. Paez D, Gnanasegaran G, Fanti S, Bomanji J, Hacker M, Sathekge $\mathrm{M}$, et al. COVID-19 pandemic: guidance for nuclear medicine departments. Eur J Nucl Med Mol. 2020;47:1615-9.

2. Skali H, Murthy VL, Al-Mallah MH, Bateman TM, Beanlands R, Better N, et al. Guidance and best practices for nuclear cardiology laboratories during the coronavirus disease 2019 (COVID-19) pandemic: an information statement from ASNC and SNMMI. J Nucl Cardiol. 2020:1-8. https://doi.org/10.1007/s12350-020-02123-2.
3. Huang HL, Allie R, Gnanasegaran G, Bomanji J. COVID19nuclear medicine departments, be prepared! Nucl.Med.Commun. 2020;41:297.

4. Mossa-Basha M, Medverd J, Linnau K, Lynch JB, Wener MH, Kicska G, et al. Policies and guidelines for COVID-19 preparedness: experiences from the University of Washington. Radiology. 2020;201326. https://doi.org/10.1148/radiol.2020201326.

5. Zhang X, Shao F, Lan X. Suggestions for safety and protection control in Department of Nuclear Medicine during the outbreak of COVID-19. Eur J Nucl Med Mol. 2020;47:1632-3.

6. Buscombe JR, Notghi A, Croasdale J, Pandit M, O'Brien J, Graham $\mathrm{R}$, et al. COVID-19: guidance for infection prevention and control in nuclear medicine. Nucl Med Commun. 2020;41:499-504.

7. Standard guideline for healthcare-associated infection control and prevention. Korean Center for Disease Control and Prevention and Korean Society for Healthcare-Associated Infection Control and Prevention. 2017. http://cdc.go.kr/CDC/cms/content/mobile/26/ 75626 view.html. Accessed 2nd Jun 2020.

8. Korean Society for Healthcare-Associated Infection Control and Prevention. Korean Center for Disease Control and Prevention. http://www.cdc.go.kr/board.es?mid=a20507020000\&bid= 0019\&act=view\&list_no=366579. Accessed 2nd Jun 2020.

9. Guidelines in response to coronavirus disease for local government. Korea Centers of Disease Control and Prevention.2020. https:// www.cdc.go.kr/board/board.es? mid=a20507020000\&bid= $0019 \&$ act $=$ view\&list no $=367279 \& \operatorname{tag}=\& n P a g e=1$. Accessed 2 nd Jun 2020.

10. Disinfection guidelines to prevent the spread of COVID-19 at public and multi-purpose facilities. Korea Centers of Disease Control and Prevention. 2020. https://www.cdc.go.kr/board/board.es?mid= a20507020000\&bid $=0019$. Acessed 15th Jun 2020.

Publisher's Note Springer Nature remains neutral with regard to jurisdictional claims in published maps and institutional affiliations. 